\title{
Time variability in the bipolar scattered light nebula of L1527 IRS: a possible warped inner disk
}

\author{
Brian T. Cook ${ }^{1}$, John J. Tobin ${ }^{2}$, Michael F. Skrutskie ${ }^{3}$, and Matthew J. Nelson ${ }^{3}$ \\ ${ }^{1}$ Leiden Observatory, 2333 CA Leiden, The Netherlands \\ e-mail: cook@strw.leidenuniv.nl \\ 2 National Radio Astronomy Observatory, Charlottesville, VA 22903, USA \\ 3 Astronomy Department, University of Virginia, Charlottesville, VA 22901, USA
}

Received 6 March 2019 / Accepted 29 April 2019

\begin{abstract}
Context. The bipolar outflows associated with low-mass protostars create cavities in the infalling envelope. These cavities are illuminated by the central protostar and inner disk, creating a bipolar scattered light nebula at near-infrared and mid-infrared wavelengths. The variability of the scattered light nebula in both total intensity and intensity as a function of position in the scattered light nebula can provide important insights into the structure of the inner disk that cannot be spatially resolved.

Aims. We aim to determine the likelihood that a warped inner disk is the origin of the surface brightness variability in the bipolar scattered light nebula associated with L1527 IRS.

Methods. We present results from near-IR imaging conducted over the course of seven years, with periods of monthly cadence monitoring. We used Monte Carlo radiative transfer models to interpret the observations.

Results. We find a time varying, asymmetrical brightness in the scattered light nebulae within the outflow cavities of the protostar. Starting in 2007, the surface brightnesses of the eastern and western outflow cavities were roughly symmetric. Then, in 2009, the surface brightnesses of the cavities were found to be asymmetric, with a substantial increase in surface brightness and a larger increase in the eastern outflow cavity. More regular monitoring was conducted from 2011 to 2014, revealing a rotating pattern of surface brightness variability in addition to a slow change of the eastern and western outflow cavities toward symmetry, but still not as symmetric as observed in 2007. We find that an inner disk warp is a feasible mechanism to produce the rotating pattern of surface brightness variability.
\end{abstract}

Key words. ISM: individual objects: L1527 - stars: formation - stars: protostars

\section{Introduction}

The formation of stars occurs in dense cores generally within molecular clouds. It is within these regions of high density where gravity overwhelms supporting forces (e.g., turbulence, magnetic fields, and thermal pressure) and the core begins to collapse. A disk is formed around a protostar during collapse due to the conservation of angular momentum and the protostar and disk work together to drive a bipolar outflow, probably through magneto-centrifugal acceleration (e.g., Blandford \& Payne 1982). The bipolar outflow then carves out conical cavities in the envelope that can be probed with observations of $\mathrm{CO}$ molecular gas (e.g., Snell et al. 1980; Arce et al. 2007), but also at near-infrared wavelengths where light from the protostar and inner disk escape through the outflow cavities and scatter on dust grains in the outflow cavity and along the cavity walls.

The system L1527 IRS is located within the Taurus molecular cloud at a distance of $\sim 140$ pc (Torres et al. 2007). It is a Class 0/I system that is characterized by its dense, presumably infalling, envelope of gas and dust (Andre et al. 1993). Its exact classification is somewhat uncertain given that it is viewed edge-on, which could make the system look younger (Tobin et al. 2008). Regardless of its exact classification, it has a well-ordered structure that has made it a prototype for studies of protostellar collapse (Ohashi et al. 1997; Sakai et al. 2014) and protostellar disk formation (Tobin et al. 2012; Ohashi et al. 2014). Within such a system, radiation from the protostar and disk is absorbed by the dusty envelope and re-emitted at longer wavelengths (Wood et al. 2001), in addition to scattering at shorter wavelengths. The shorter wavelength scattering gives rise to scattered light nebulae that can be prominent toward protostars at near to mid-infrared wavelengths (e.g., Seale \& Looney 2008).

Scattered light nebulae have long been used to discern the behavior of the emitting object; an early example is Hubble's work on NGC 2261 (also known as R Mon or Hubble's Variable Nebula, Hubble 1917). These scattered light images can complement millimeter studies of more optically thin dust emission in order to probe the envelope and disk structure of protostars and pre-main sequence stars (Guilloteau \& Dutrey 2008; Wolf et al. 2008; Tobin et al. 2012; Segura-Cox et al. 2016; Sheehan \& Eisner 2017); scattered light images on envelope scales can also show effects due to changes of the illumination source, the inner disk, and protostar at the heart of the system (Tobin et al. 2008; Connelley et al. 2009).

The inner disk structure can affect the illumination and brightness of the outflow cavities because the inner disk rim emits the majority of photons at wavelengths that can be detected in scattered light for protostars (Whitney et al. 2003; Muzerolle et al. 2003). A natural cause of variability in the scattered light nebulae could be a rotating warped disk, and astrophysical disks are often warped. This occurs when an initially planar disk is misaligned to a component of the potential (e.g., the orbit of a 
Table 1. L1527 observation log.

\begin{tabular}{llllll}
\hline \hline $\begin{array}{l}\text { Date } \\
(\mathrm{UT})\end{array}$ & Telescope/Instrument & $\begin{array}{l}\text { Integration time } \\
(\mathrm{min})\end{array}$ & $\begin{array}{l}\text { Airmass } \\
(\mathrm{sec}(z))\end{array}$ & $\begin{array}{l}\text { Seeing } \\
\left({ }^{\prime \prime}\right)\end{array}$ & $\begin{array}{l}\text { Standard deviation } \\
\left(\mathrm{MJy} \mathrm{sr}^{-1}\right)\end{array}$ \\
\hline 2007 December 28 & Hiltner 2.4 m/TIFKAM & 35 & 1.03 & 1.0 & 0.0561 \\
2009 December 03 & Hiltner 2.4 m/TIFKAM & 30 & $1.06-1.16$ & 0.9 & 0.0446 \\
2011 October 07 & 31 in/FANCAM & 65 & $1.03-1.1$ & 1.3 & 0.0298 \\
2011 October 23 & 31 in/FANCAM & 95 & $1.24-1.04$ & 1.2 & 0.0262 \\
2011 November 07 & 31 in/FANCAM & 100 & $1.3-1.05$ & 1.2 & 0.0276 \\
2011 December 11 & 31 in/FANCAM & 100 & $1.2-1.05$ & 2.0 & 0.256 \\
2012 January 07 & 31 in/FANCAM & 115 & $1.28-1.03$ & 2.25 & 0.0255 \\
2012 January 31 & 31 in/FANCAM & 100 & $1.09-1.04$ & 1.75 & 0.0307 \\
2012 February 18 & 31 in/FANCAM & 105 & $1.02-1.15$ & 1.8 & 0.0258 \\
2012 March 02 & 31 in/FANCAM & 70 & $1.16-1.46$ & 1.75 & 0.0364 \\
2012 March 15 & 31 in/FANCAM & 140 & $1.1-2.2$ & 1.5 & 0.0328 \\
2013 January 21 & 31 in/FANCAM & 110 & $1.11-1.03$ & 1.75 & 0.0455 \\
2013 February 21 & 31 in/FANCAM & 110 & $1.02-1.18$ & 2.5 & 0.0310 \\
2013 April 14 & 31 in/FANCAM & 50 & $1.65-2.4$ & 2.0 & 0.0343 \\
2013 April 21 & 31 in/FANCAM & 50 & $1.95-3.3$ & 2.5 & 0.0334 \\
2013 October 02 & 31 in/FANCAM & 95 & $1.17-1.02$ & 1.25 & 0.0250 \\
2013 November 04 & 31 in/FANCAM & 105 & $1.24-1.03$ & 1.8 & 0.0242 \\
2013 December 19 & 31 in/FANCAM & 105 & $1.22-1.03$ & 1.4 & 0.0224 \\
2014 January 20 & 31 in/FANCAM & 130 & $1.15-1.02$ & 2.8 & 0.0230 \\
2014 February 28 & 31 in/FANCAM & 105 & $1.02-1.2$ & 2.0 & 0.0285 \\
\hline
\end{tabular}

companion star), or when the disk becomes unstable to tilting by tidal (Lubow 1992) or radiation effects (Maloney et al. 1996; Nixon \& King 2016). In Watson \& Stapelfeldt (2007), the asymmetry of HH 30's circumstellar disk is explored. They found that the brightness of the upper nebula could vary by a factor of 0.2 mag in just two days. While analyzing the habitable zone of T Tauri stars in the mid-infrared, Ke et al. (2012) postulate that a magnetized and misaligned host star can cause a periodic warp that in turn leads to a periodic modulation of the spectral energy distribution. The provided explanation for a warp of this kind is either a companion star or a dynamic interface between the star's magnetic field and the disk, resulting in a variable scale height.

The variability of L1527 IRS was first recognized in multiepoch images from the Spitzer Space Telescope (Tobin et al. 2008) and it has been further characterized in ground-based monitoring conducted over the course of several years. Section 2 outlines the observations of L1527 IRS from 2007 to 2014, while Sect. 3 is devoted to discussing the observed characteristics of the outflow cavities. We explore the possible origin of the scattered light variability by introducing changes to the inner disk structure in Sect. 4 in an effort to qualitatively match the observations and provide insights into the small-scale structure of the protostellar disk. We discuss our results in Sect. 5 and present our conclusions in Sect. 6.

\section{L1527 observations}

The observations were conducted with the FanCam near-infrared camera on the 31 inch Tinsley reflector operated by the University of Virginia and located at Fan mountain near Charlottesville, Virginia. The camera features an $8.7^{\prime} \times 8.7^{\prime}$ field of view on a $1024 \times 1024$ Teledyne Imaging Sensors HAWAII- 1 detector array, and was designed for use from $\sim 1$ to $2.5 \mu \mathrm{m}$ (Kanneganti et al. 2009). A small telescope with infrared instrumentation is capable of observations over several years, which is not as easily achieved at larger observatories. Thus, the FanCam camera was ideal for these observations. L1527 IRS was observed on multiple nights from Fall 2011 to Spring 2014 (see Table 1). We also include previous imaging from the TIFKAM instrument on the $2.4 \mathrm{~m}$ Hiltner telescope at the $\mathrm{MDM}^{1}$ observatory in 2007 (Tobin et al. 2008), and an additional observation from MDM in December 2009 is also presented here.

The data were reduced using standard methods for groundbased near-infrared imaging, using the upsqiid package of the Image Reduction and Analysis Facility (IRAF). We used twilight sky flat fields or skyflats constructed from the observations. The FanCam observations were conducted in a ten-point dither pattern with L1527 IRS centered on one quadrant of the detector for each dither pattern, rotating through the quadrants after each complete dither pattern. The centering of L1527 IRS in different quadrants for each dither pattern enabled the preceding and following dither patterns to be median combined to produce a sky intensity image that was then subtracted from the data. Each integration was $30 \mathrm{~s}$ and the flat-fielded, sky-subtracted images were median combined to produce a single mosaic. Often the final mosaic image from a single night of observations consists of 100-200 individual frames. The frames were aligned by centroiding on a star that was present in all observations. Tobin et al. (2008) describe the data reduction process with upsqiid in more detail. We observed primarily in the $K s$-band filter because the scattered light nebula of L1527 is best detectable at this wavelength in ground-based observations.

\section{Results}

The near-infrared images toward L1527 IRS are shown in Fig. 1. The outflow cavities of L1527 IRS are oriented in almost exactly the east-west direction. The system is also oriented with the equatorial plane of the disk viewed nearly edge-on (Tobin et al. 2008, 2010), making the outflow cavities on both sides of the

\footnotetext{
1 Originally named the Michigan-Dartmouth-MIT observatory, the acronymn is now the standard name as various institutions have joined or left the consortium.
} 
B. T. Cook et al.: Time variability of L1527 IRS

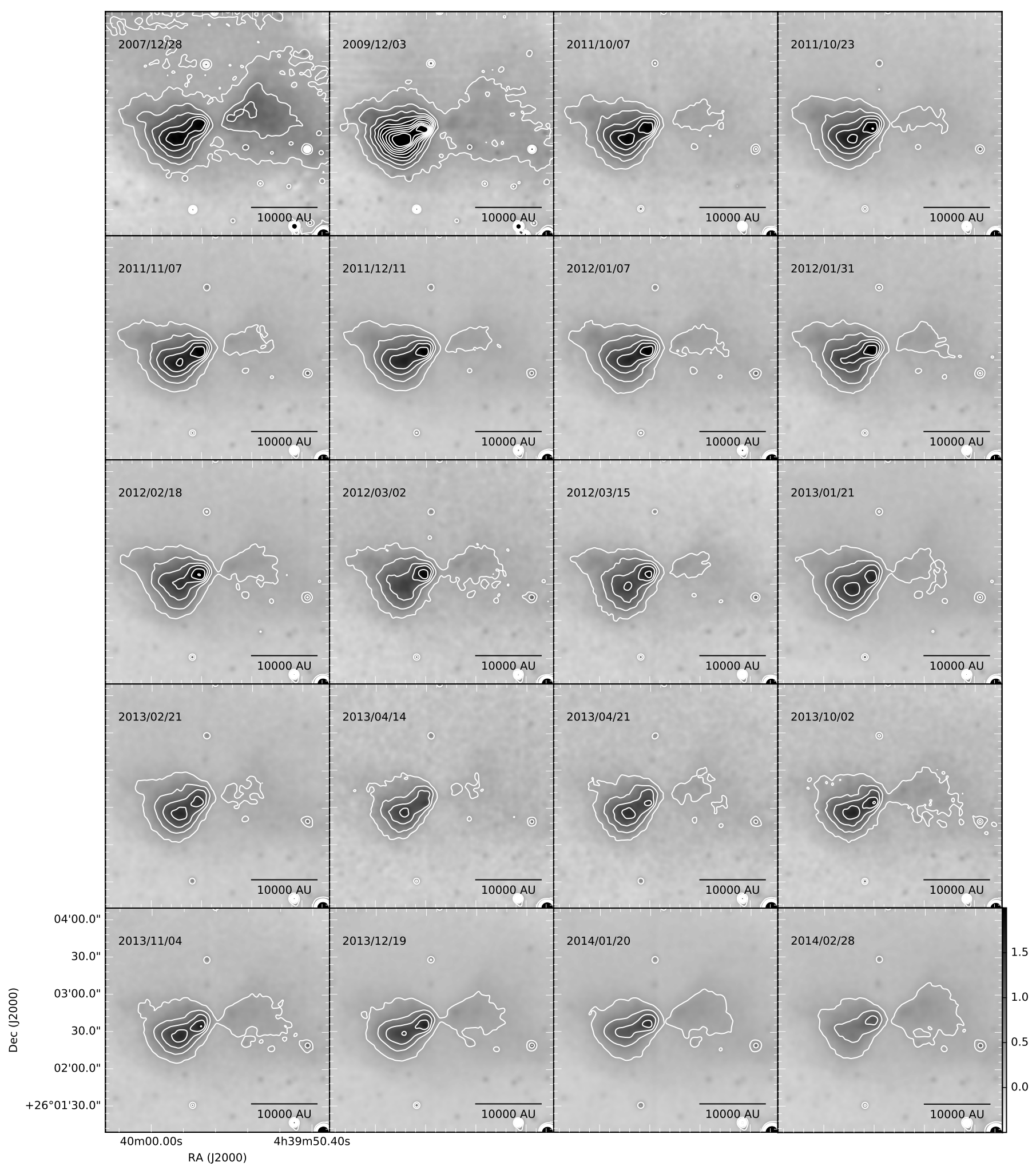

Fig. 1. Observations of L1527 in Ks-band from December 2007 to April 2014 shown as inverse gray scale. The contour levels are $3.5 \times n$ where $n$ is the set of numbers $0.1,0.2, \ldots, 1.0$. As stated in Sect. 3, the primary features of the scattered light nebulae during this observation run are the western lobe's emergence and the gradual transition toward a more symmetric brightness distribution of the cavities. The color bar shown in the lower right panel is in units of $\mathrm{MJy} \mathrm{sr}^{-1}$.

protostar visible through comparable amounts of extinction, but the eastern side of the outflow cavity remains brighter throughout our observations. Only in the 2007 observation and final observation in 2014 do they appear at nearly equal brightness, as shown in Fig. 2. It is still not completely clear which side of the outflow cavity is tilted toward our line of sight. For a spherical envelope, one would assume that the brighter outflow cavity would be tilted toward us, and the upper layers of the disk 


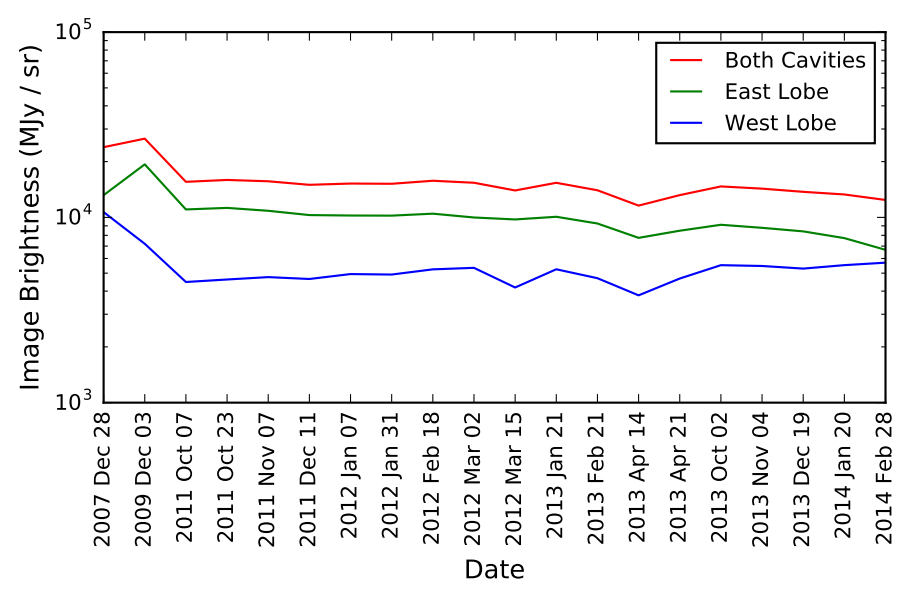

Fig. 2. Image brightness as a function of time, indicating that the eastern lobe is indeed brighter for the entire observational run.

from Tobin et al. (2010) show the eastern disk surface to appear brighter at $3.8 \mu \mathrm{m}$. There is also substantial overlap between red and blue-shifted $\mathrm{CO}$ emission on either side of the outflow cavity (Bontemps et al. 1996). However, by modeling the envelope kinematics Oya et al. (2015) suggest that the western outflow cavity is tilted slightly toward us, but only by about $5^{\circ}$.

The first two observations from 2007 and 2009 in Fig. 1 are substantially different, which, along with the variability detected with Spitzer in Tobin et al. (2008), establishes the variability of the protostellar system and feasibility of characterizing it with ground-based observations. However, as these observations took place several years before the rest (and two years apart), it is difficult to place them within the context of any periodic phenomena of L1527 IRS. The surface brightness of the eastern lobe in 2009 is significantly greater than at later times. When monthly observations began in October 2011, the overall intensity decreased to $\sim 70 \%$ of the 2007 brightness and steadily decreased from 2011 to 2014 .

The evolution of the western lobe is more peculiar than that of the eastern lobe. In 2009 and 2011, it is only marginally detected and remains faint through 2012 and early 2013. The brightness of the western lobe began to increase in April 2013 and remained in a brighter state through the end of observations in early 2014, but it never had a higher surface brightness than the eastern lobe.

To more clearly illustrate the variation in the scattered light nebula, we show the observations of the protostellar system with the average of all the observed images subtracted in Fig. 3. We find that a pattern of variable illumination is strongly present in the eastern lobe and the areas of greater than average illumination (and less than average) appear to rotate across the scattered light nebulae from south to north with increasing time. This is in the same direction as the disk and envelope rotation observed by Ohashi et al. (1997) and Tobin et al. (2012). The rotating character of the illumination is not evident in the western lobe, probably due to its overall low intensity. During 2013 and 2014, the overall intensity of the eastern lobe was lower, causing an overall deficit of emission when the average is subtracted, but we can still see areas of enhanced and depressed illumination rotating across the eastern lobe.

\section{Radiative transfer modeling}

The variable illumination and its apparent rotational nature suggest a connection with the illumination source, which is primarily the inner disk and protostar. Thus, the origin of the variability may be related to the inner disk structure and we conducted radiative transfer modeling to examine the possible effects of the inner disk that may present themselves as variable illumination in the scattered light nebula. We used the radiative transfer code of Whitney et al. (2013), for which previous versions of the code (Whitney et al. 2003) have been used to model L1527 IRS (Tobin et al. 2008, 2010, 2013). The code produces images in the near-infrared by propagating photons from the central star, accretion hotspots, and disk that can either be absorbed or scattered on their way from the protostar and disk out into the envelope. While the code can also produce spectral energy distributions, our focus here is on visualizing the effects of the disk on the overall scattered light images.

The code now includes the ability to add perturbations to the disk in the form of gaps, spirals, warps, and/or a puffed-up inner rim. Furthermore, a misaligned inner disk, with respect to the outer disk, is also possible. Since most of the emission in the scattered light nebula emanates from the inner, opticallythick rim of the dusty disk, we used a warped inner disk to compare with the observations. The remaining parameters are kept unchanged from Tobin et al. (2013) as we modify the inner disk only. From there, we produced images at different azimuthal viewing angles in $15^{\circ}$ increments to observe how the illumination of the scattered light nebula changed with the rotation of the warped inner disk.

The structure of the inner disk warp is visualized in Fig. 4 where we computed a model without an envelope, focusing on very small radii to resolve the inner disk. The warped structure is rather narrow and extends very high vertically from the disk plane. The warp manifests as a variable scale height $h(\varphi)$ dependent on azimuthal angle such that $h(\varphi)=h_{\max } \cos ^{n} \varphi$, where $h_{\text {max }}$ is the maximum height (see Table 2, defined to occur at disk azimuthal angle $\varphi=0$ ) and $n$ is the warp exponent. We note that our assumed warp parameters are probably not physically realistic, but the exaggerated warp is meant to demonstrate the feasibility of a warp to produce the variable illumination. We found that warps with a large exponent (odd integers between 39 and 47, see Table 2) were the ones that best provided the type of illumination pattern within the outflow cavities that could be consistent with the observations. We note, however, that we did not fully explore the parameter space, so the parameters of the warp we use in this paper are by no means a fit to the data. They simply demonstrate the same physical behavior as observed toward L1527 IRS and it is likely that other warp parameters could produce similar results with sufficient exploration of parameter space.

Figure 5 shows the effect of this warp on the simulated scattered light nebula at larger scales, approximately the same scale from which L1527 IRS is viewed in the FanCam observations, as shown in Fig. 1. We view the system in increments of $15^{\circ}$ in the $\phi$ direction (azimuthal coordinate) to mimic the effect of the inner disk rotating.

At $\phi=0$ to $45^{\circ}$ of Fig. 5 (top row) the scattered light morphology is approximately symmetric and there is no change in the illumination; the same is true for $\phi=135-180^{\circ}$ (bottom row). However, for $\phi=60-120^{\circ}$ (middle row of Fig. 5), there is a clear slant in the scattered light images from the southeast to northwest. Furthermore, we did not simulate $\left(\phi=195-360^{\circ}\right)$ since the illumination pattern would be mirrored. Thus, on average this illumination pattern would show a rotating pattern of increased brightness, which is reflected in Figs. 6 and 7, which show the same simulation outputs at a smaller scale without smoothing or with the average subtracted out, respectively. The latter shows 


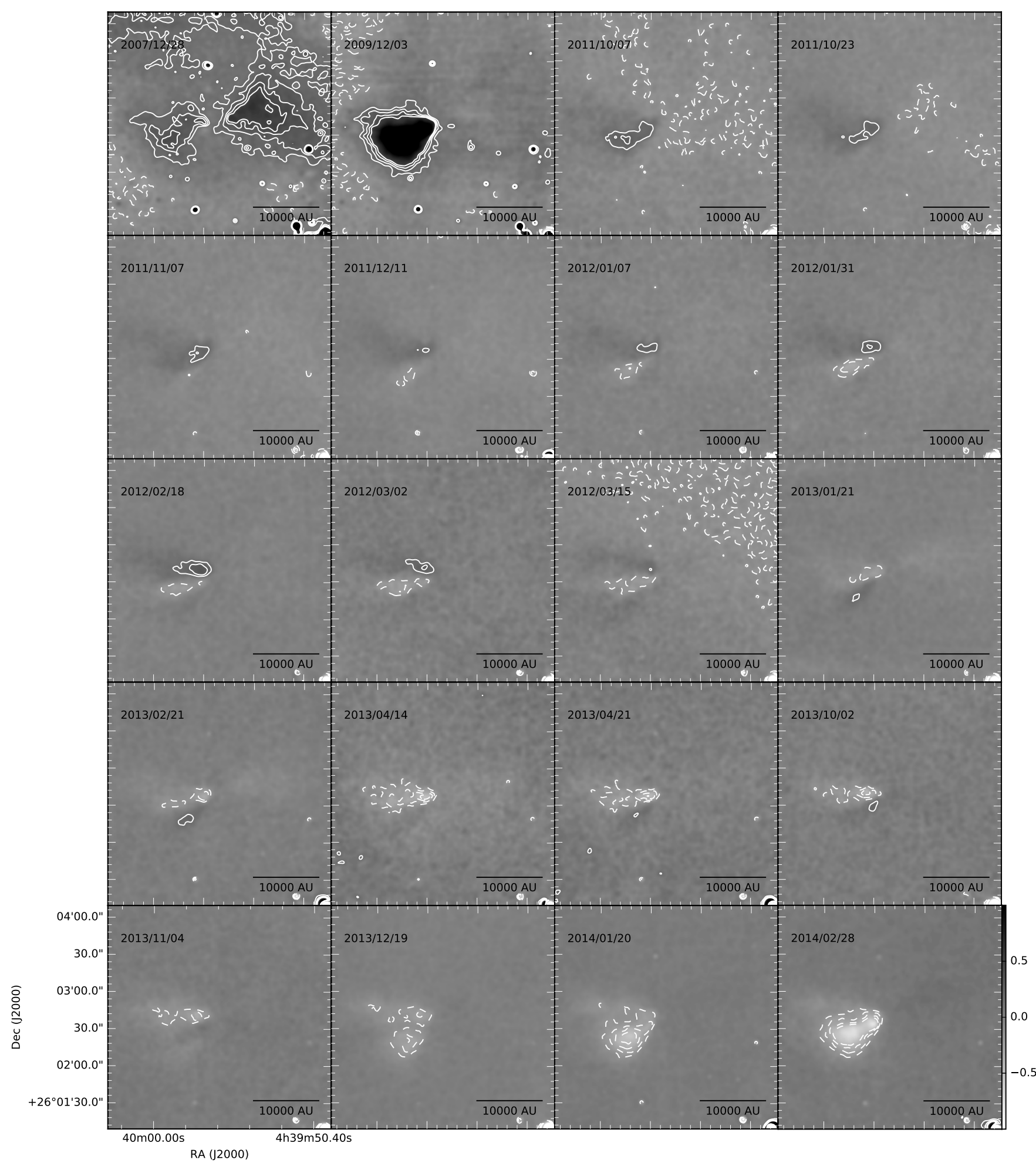

Fig. 3. Observations with the average image of L1527 subtracted out, December 2007-April 2014 displayed as inverse grayscale. The contour levels are $-0.45,-0.35,-0.25,-0.15,0.15,0.25,0.35,0.45$. Black areas are regions where the emission is brighter than the average and white areas are regions where the emission is fainter than the average. In this set of images the rotational character of the eastern lobe becomes more clear as the black and white flip from December 2011 to February 2013. The color bar shown in the lower right panel is in units of $\mathrm{MJy} \mathrm{sr}^{-1}$.

that there is a correlation between intensity and the shearingtype action on larger scales. We do not attempt to fully reproduce the asymmetric intensity of the eastern and western lobes or the overall decrease of intensity. However, this result demonstrates that a warp in the inner disk can produce a rotating pattern of illumination variability.

\section{Discussion}

The clear morphological variability of the outflow cavities in the observations and the apparent rotational nature of the variability strongly suggests that the origin of the variable illumination is from the disk surrounding the protostar. The disk has been 


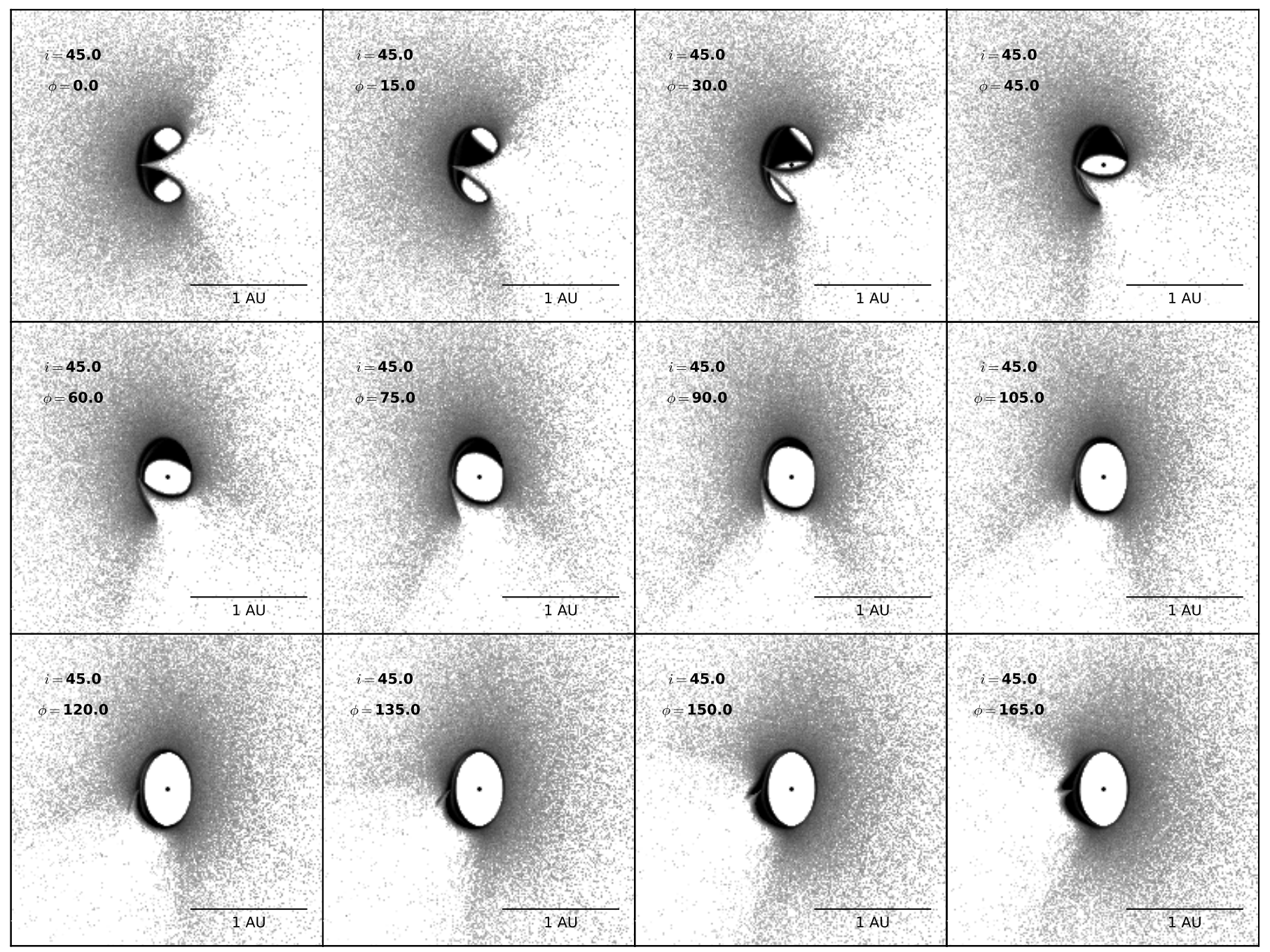

Fig. 4. View of the warped inner disk from the radiative transfer model at $K s$-band from an inclination angle of $45^{\circ}$ better shows the structure. The image is plotted as an inverse grayscale where darker means more intensity, thus the white triangular region that rotates between images corresponds to shadowing by the inner disk warp.

observed at multiple millimeter and sub-millimeter wavelengths (Maury et al. 2010; Tobin et al. 2012, 2013; Ohashi et al. 2014; Aso et al. 2017; van 't Hoff et al. 2018), finding clear evidence of a dusty, rotationally supported disk. However, the edge-on nature of the system, and high opacity of the midplane prevent us from studying the nature of the inner disk with millimeter interferometry. The likely sub-AU scales of the dust destruction radius, where most of the emission of the scattered light cavities in L1527 IRS originates, is still beyond the reach of observatories like the Atacama Large Millimeter/submillimeter Array (ALMA) and the Very Large Array (VLA), even in the most nearby systems (Andrews et al. 2016). Thus, observations of protostellar variability provide some of the only hints of the inner disk structure. Many protostars are shown to have variability in their near to mid-infrared photometry from the Young Stellar Object VARiability (YSOVAR) survey (Morales-Calderón et al. 2011; Rebull et al. 2015). Several Class 0 systems were found to exhibit modest variability by the YSOVAR survey, and at first glance the variable character of L1527 IRS may appear different due to the variability being resolved in the scattered light cavities out to $\sim 0.1 \mathrm{pc}$ scales. However, YSOVAR was observing more distant star-forming regions, so it is possible that variability in the scattered light cavities was occurring, but could not be resolved by Spitzer.

\subsection{Possibility of the variability originating from the inner disk}

The variability in L1527 is consistent with having an inner disk origin as demonstrated with a disk warp model showing a variable illumination pattern. The central protostellar mass of L1527 is known to be $0.2-0.45 M_{\odot}$ from observations of molecular line kinematics from the disk (Tobin et al. 2012; Ohashi et al. 2014; Aso et al. 2017; Sakai et al. 2014). Our sparse time sampling, with significant gaps, makes it difficult to ascertain if (or when) we have observed a complete rotation period of the variable illumination. We can see that there was a bright limb on the southeast portion of the nebula when we started monitoring in October 2011 and then this feature is again present at the same location in January 2013. If we assume that this motion accounts for approximately one orbital period, the length of time is $\sim 15$ months or more simply 500 days. If the scattered light variability is related to the rotation period of the inner disk, Kepler's third law indicates that an orbital period of $\sim 480$ days around a $0.35 M_{\odot}$ protostar corresponds to $\sim 0.85 \mathrm{AU}$. This points 
Table 2. Warped disk model parameters, most of which have been unchanged from the best fit model in Tobin et al. (2013).

\begin{tabular}{|c|c|c|}
\hline Parameters & Description & Values \\
\hline$\gamma^{\prime} \mathrm{s}$ & Number of photons & $1 \times 10^{8}$ \\
\hline$R_{\star}\left(R_{\odot}\right)$ & Stellar radius & 2.09 \\
\hline$T_{\star}(\mathrm{K})$ & Stellar temperature & 4000 \\
\hline$M_{\star}\left(M_{\odot}\right)$ & Stellar mass & 0.5 \\
\hline$\hat{M_{\text {disk }}}\left(M_{\odot}\right)$ & Disk mass & 0.005 \\
\hline$h(100 \mathrm{AU})$ & Disk scale height at $100 \mathrm{AU}$ & 48.0 \\
\hline$H_{0}\left(R_{\star}\right)$ & Disk scale height at $R_{\star}$ & 0.03 \\
\hline$\alpha$ & Disk radial density exponent & 2.5 \\
\hline$\beta$ & Disk scale height exponent & 1.3 \\
\hline$\dot{M}_{\text {disk }}\left(M_{\odot} \mathrm{yr}^{-1}\right)$ & Disk accretion rate & $1.5 \times 10^{-6}$ \\
\hline$R_{\text {trunc }}\left(R_{\star}\right)$ & Magnetosphere co-rotation radius & 3.0 \\
\hline$F_{\text {spot }}$ & Fractional area of accretion hotspot & 0.01 \\
\hline$R_{\text {disk,min }}\left(R_{\star}, \mathrm{AU}\right)$ & Disk inner radius & $14.25,0.14$ \\
\hline$R_{\text {disk,max }}(\mathrm{AU})$ & Disk outer radius & 125 \\
\hline$R_{\text {env, min }}\left(R_{\star}\right)$ & Envelope inner radius & 42.75 \\
\hline$R_{\mathrm{env}, \max }(\mathrm{AU})$ & Envelope outer radius & 15000 \\
\hline$\dot{M}_{\text {env }}\left(M_{\odot} \mathrm{yr}^{-1}\right)$ & Envelope mass infall rate & $4.5 \times 10^{-6}$ \\
\hline$\rho_{1 \mathrm{AU}}\left(\mathrm{g} \mathrm{cm}^{-3}\right)$ & Envelope density at $1 \mathrm{AU}$ & $7.25 \times 10^{-14}$ \\
\hline$b_{\text {out }}$ & Outer cavity shape exponent & 1.5 \\
\hline$\theta_{\text {open,out }}\left({ }^{\circ}\right)$ & Outer cavity opening angle & 20 \\
\hline$\rho_{c}\left(\mathrm{~g} \mathrm{~cm}^{-3}\right)$ & Cavity density & 0 \\
\hline$\beta_{\text {dust,mm }}$ & Millimeter dust spectral index & 0.25 \\
\hline Warp height & Multiplicative factor of warp scale height above disk scale height & 10.0 \\
\hline$n$ & Exponent for azimuthal disk warp $\left(\cos ^{n}\right)$ & 41 \\
\hline$\theta\left({ }^{\circ}\right)$ & Inclination angles of high $\mathrm{S} / \mathrm{N}$ images & 85.0 \\
\hline$\phi\left({ }^{\circ}\right)$ & Azimuthal angles & $0,15.0,30.0, \ldots, 180.0$ \\
\hline$\lambda(\mu \mathrm{m})$ & wavelength & 2.15 \\
\hline
\end{tabular}

Notes. Parameters most relevant to this result are in boldface and parameters that have been adjusted from the value in the best fit model are italicized. The stellar mass is only used in setting the density structure, changing this to the measured protostar mass would require increasing the envelope infall rate to maintain the same density structure.

to an origin of variable illumination in the inner disk at a distance of $\sim 0.85 \mathrm{AU}$ from the protostar, which is roughly comparable to the radius of the inner disk (see Table 2) from which the proposed warp originates. The dust destruction radius, where the optically thick inner disk begins, is typically defined to be at a temperature of $\sim 1400 \mathrm{~K}$. For L1527, the inner radius of the dusty disk is expected to be $\sim 0.15 \mathrm{AU}$ if the dusty disk extends all the way to the dust destruction radius. However, our estimate of the orbital period has significant uncertainty in that it could be shorter (or longer) than we are suggesting here. However, in any event the timescale for the morphological variability at least appears consistent with the orbital timescales expected to be found in the inner disk.

\subsection{Other possible mechanisms for variability}

While we did not explore all possibilities to produce the observed variability within the radiative transfer models, there are other effects beyond a disk warp that could also give rise to variability. One possibility that can also produce an asymmetric brightness distribution in the outflow cavities is a misaligned inner disk. Matsumoto et al. (2017) suggested that the misalignment of a protostellar disk could arise from the acceleration of the outflows due to a magnetic field. If the protostar within L1527 IRS is rotating rapidly, then the star itself will be somewhat oblate, causing the spin axis of the star to trail that of the disk, which could cause a disk misalignment (Spalding et al. 2014). Disk tilting would also cause stochastic wandering of the bipolar outflows. Depending on the extent of the misalignment, a portion of the inner disk beyond the misaligned portion would be shadowed and a portion would be more highly illuminated. Wood et al. (2001) showed that misaligned disks in a close binary system could preferentially illuminate one side of the outflow cavity, but in this case the misaligned inner disk of an assumed single system could produce the same effect and the brighter portion of the inner disk beyond the misaligned disk could give rise to the rotating pattern of the scattered light variations that might have a period of $\sim 1.3 \mathrm{yr}$. However, this may require a somewhat fine-tuned system.

Several other mechanisms can result in a misaligned inner disk, most of which are related to planet-disk interactions, or possibly companion star-disk interactions. Thus, misaligned inner disks are observed toward gapped protoplanetary disks (e.g., AA Tau; Loomis et al. 2017) and others show shadowing in high-resolution observations of disks that can be attributed to an inner disk (Zhu 2019). It is unclear whether L1527 IRS has a gapped disk (due to a planet or unseen companion) and how far that gap could extend and still produce a significant amount of scattered light. A misaligned inner disk alone could not produce an asymmetric illumination pattern because it would require an extremely large misalignment angle, and the precession of the misaligned inner disk would have to have a timescale on the order of the $\sim 1.3 \mathrm{yr}$ possible period of the rotating illumination pattern. Misaligned inner disks are shown to precess 


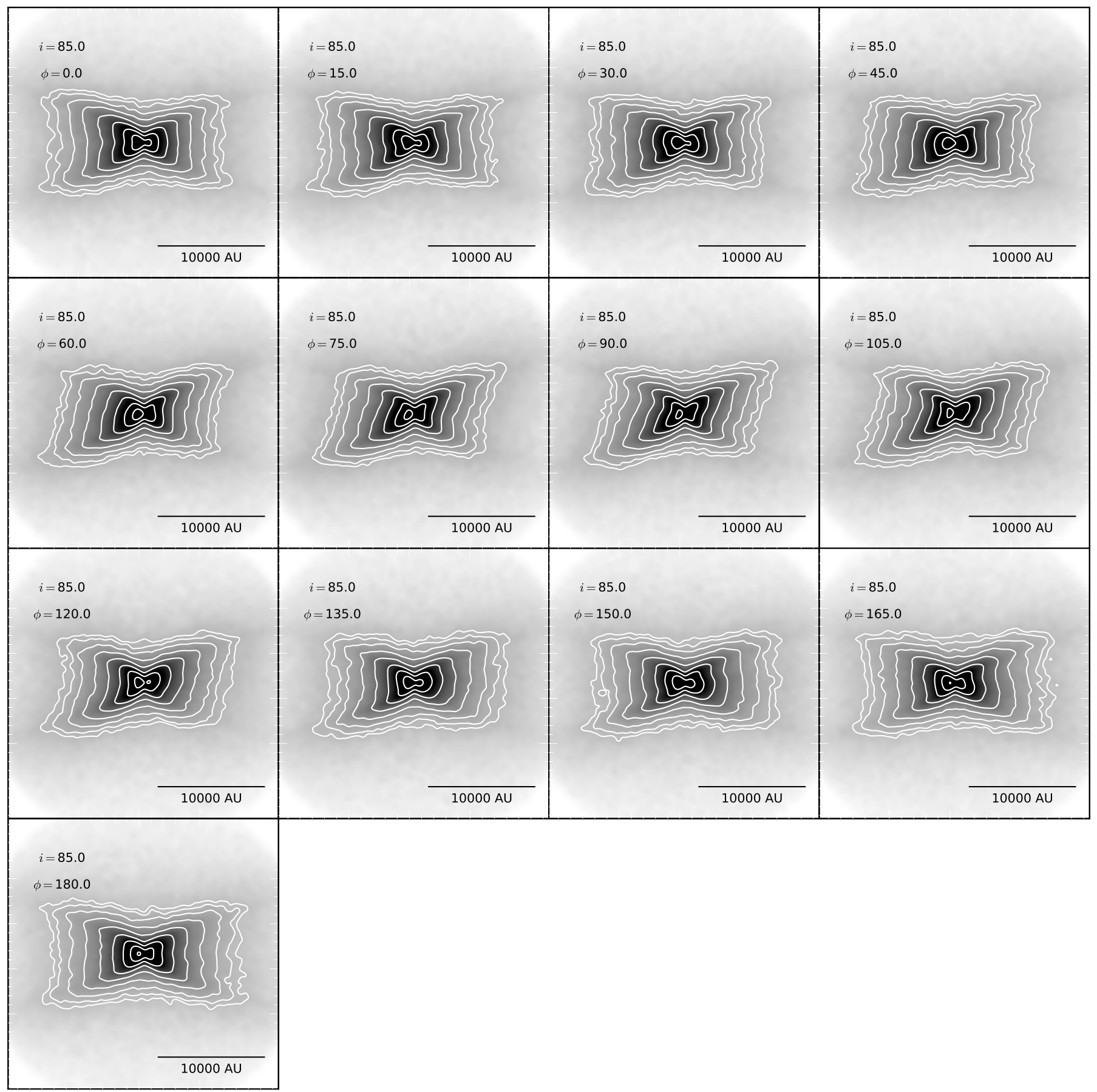

Fig. 5. Disk warp simulations viewed on the scale of the protostellar envelope, similar to the field of view shown for L1527 IRS in Fig. 1. These images viewed in $15^{\circ}$ increments of azimuthal angle show the effect of a rotating inner disk warp on the scattered light nebula on larger scales. The contour levels are defined as $n=2^{m}$, where $m$ is the set of integers from -2 to 6 and the units are $\mathrm{MJy} \mathrm{sr}^{-1}$.

on greater than $100 \mathrm{yr}$ timescales from numerical simulations (Facchini et al. 2018).

It remains possible that there is an unseen binary companion passing above and below the disk plane on a short period orbit. This companion could produce an asymmetric illumination pattern in the outflow cavities as well as partially explain the periodic behavior seen in the observations. Furthermore, an unseen binary companion could also lead to the possible production of a misaligned inner disk and/or a warp in the disk. Thus, a combination of multiple effects could be combined to produce the overall morphology of the outflow cavity illumination and an unseen companion could naturally produce many of these effects, as has been shown in numerical models (e.g., Zhu 2019). However, it is unclear if such a close companion could be observationally verified in the near future. The disk midplane is completely obscured in the near to mid-infrared (Tobin et al. 2010), and the dust continuum emission is optically thick in the submillimeter and millimeter (van 't Hoff et al. 2018), effectively hiding any potential companion. Moreover, VLA observations at $7 \mathrm{~mm}$ are the highest resolution possible at present for more optically thin dust emission and these data only show a disk and do not have the resolution to examine structures on $\sim 1 \mathrm{AU}$ scales 


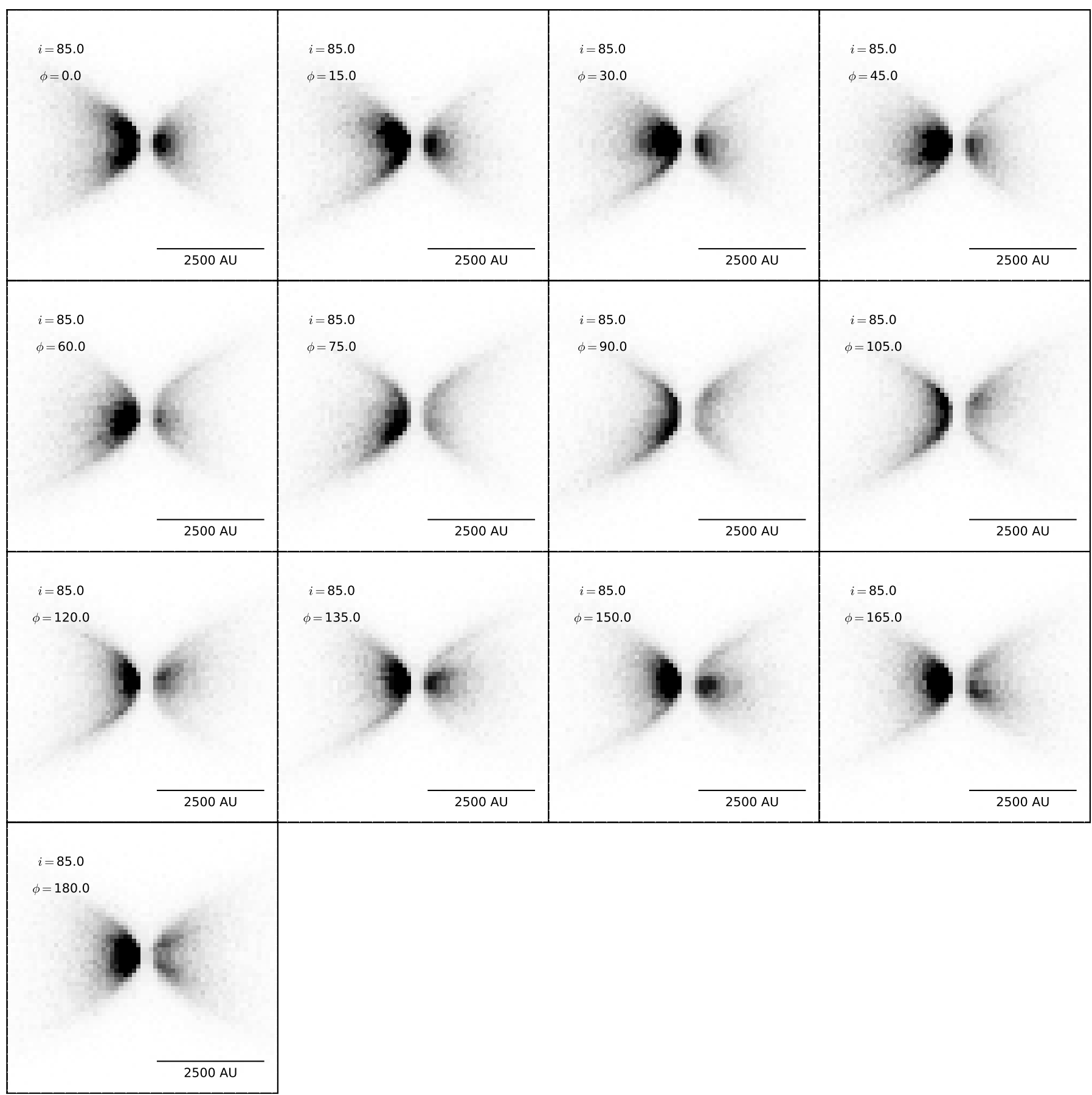

Fig. 6. Disk warp simulations viewed on a zoomed-in scale compared to Fig. 5 without smoothing. These images viewed in $15^{\circ}$ increments of azimuthal angle show the effect of a rotating inner disk warp on the scattered light nebula on smaller scales.

(Melis et al., in prep.). The enhanced Multi Element Remotely Linked Interferometer Network (e-MERLIN) could be used for higher resolution, but only if L1527 IRS is bright (and compact) enough at those resolutions.

\subsection{Additional examples of scattered light variability}

System L1527 IRS is just one example of what may be a class of objects showing resolved time variability in their scattered light nebulae. One of the original variable scattered light nebulae from R Mon (also known as Hubble's Variable Nebula, Hubble 1917) is also found around a young, but massive star
(Fuente et al. 2006). There has also been periodic variability observed in the protostar Per-emb-28 (LRLL 54361), which was attributed to a binary interaction resulting in pulsed accretion on an $\sim 25$ day period (Muzerolle et al. 2013). However, there are other examples toward more similar low-mass protostars that are more similar to the slow changes observed in L1527 IRS.

System L483 IRS as reported by Connelley et al. (2009) is the most directly comparable system to L1527 IRS. It is also a Class 0/I protostar viewed nearly edge-on and both cavities are visible. L483 IRS has similar variability characteristics to L1527 IRS as well. It has resolved morphological variability, variability in overall amplitude, and independent variations in 


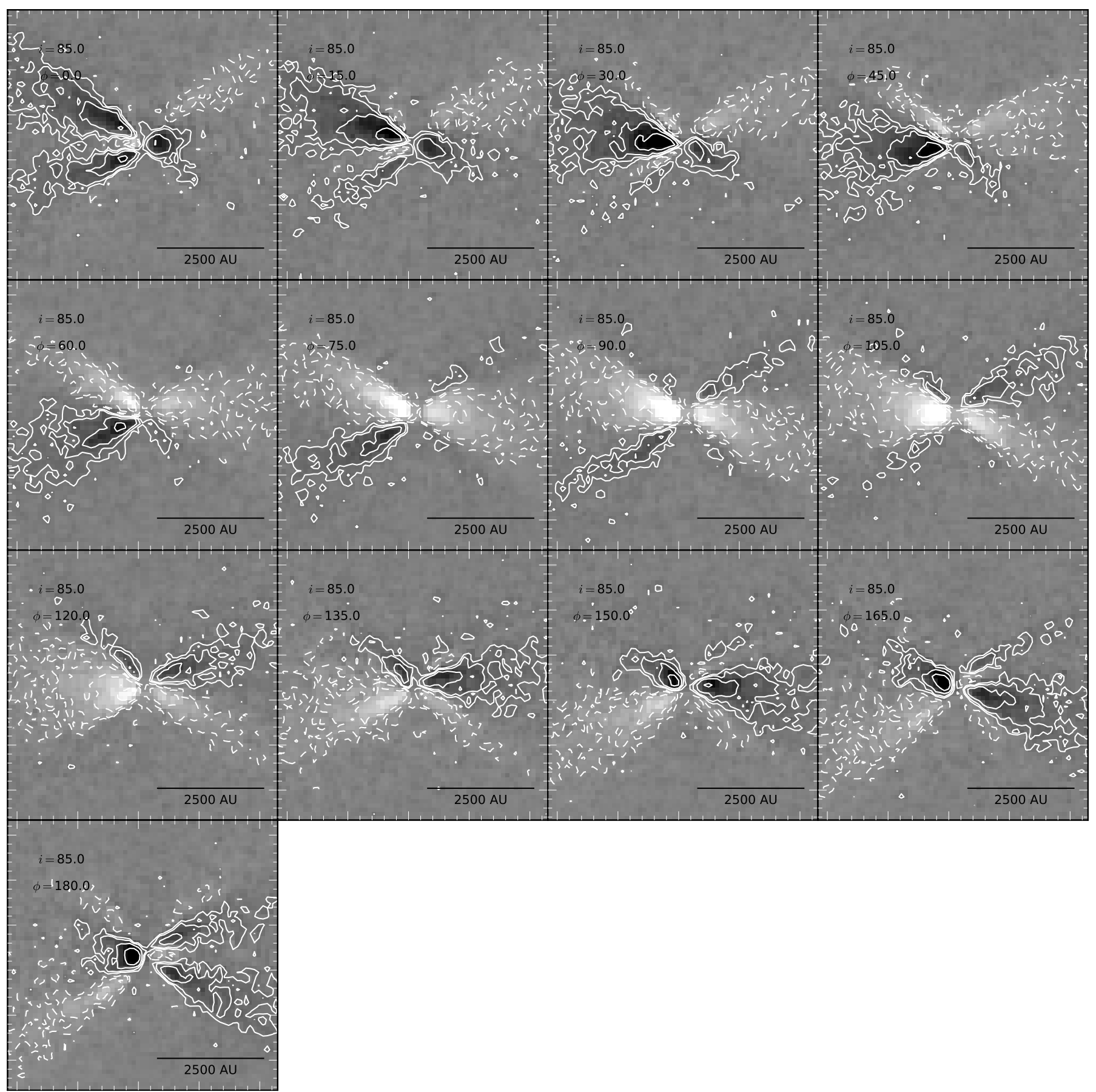

Fig. 7. Disk warp simulations viewed on a zoomed-in scale compared to Fig. 5 with the average subtracted out. These images viewed in $15^{\circ}$ increments of azimuthal angle show the effect of a rotating inner disk warp on the scattered light nebula on smaller scales. The contour levels are $\pm 2^{m}$, where $m$ is the set of integers from 0 to 3 and the units are $\mathrm{MJy} \mathrm{sr}^{-1}$.

the bipolar scattered light cavities. However, L483 IRS has not been subject to the more frequent monitoring that we conducted for L1527 IRS; the observations of L483 are spread over many months to years apart. Connelley et al. (2009) suggested opaque clouds within $\sim 1 \mathrm{AU}$ of the protostar could be the cause of the variability, but such small-scale features could also be associated with an inner disk.

The behavior of L1527 IRS is also analogous to the disk around $\mathrm{HH} 30$, but $\mathrm{HH} 30$ shows clear variations in scattered light disk rather than the large-scale nebula. Watson \& Stapelfeldt (2007) discuss many possible mechanisms to produce the variation, including variation that only affects one side of the nebula.
They conclude that a combination of overall variability and disk asymmetries (including a possible warp) could be responsible for the variability in the scattered light disk.

We also mentioned the YSOVAR studies that find numerous variable protostellar and pre-main sequence stars with disks that show variability with a variety of light curves (Morales-Calderón et al. 2011). These studies could be revealing the types of inner disk variability that might give rise to the morphological variability in the scattered light nebula toward L1527 IRS and other protostars. L1527 IRS and L483 IRS are not likely to be the only examples of variable scattered light nebulae, and the data already exist to enable further discovery of these types of objects 
using archival Spitzer and Wide-field Infrared Survey Explorer data.

\section{Conclusions}

We have presented ground-based near-infrared monitoring of the Class 0 protostar L1527 IRS finding variability within its scattered light nebula through observations at the $K s$-band taken over the course of seven years, and higher cadence monitoring that was conducted over three years. We find variability in the overall surface brightness of the scattered light nebula from the protostar, independent variability in both of the bipolar outflow cavity lobes, and variability in the illumination pattern of the cavities that appears to rotate across the eastern outflow cavity. We have demonstrated that an inner disk warp can produce a variable pattern that would rotate across the outflow cavity as the inner disk orbits the protostar.

Other mechanisms such as a misaligned inner disk and/or an unseen binary companion could also possibly produce the observed variable illumination in the scattered light nebulae, but we aimed to produce the effect with a minimal set of parameters. Higher and more uniform cadence monitoring with a larger telescope with better seeing could lead to an improved characterization of the variability in L1527. However, regardless of the details, we conclude that the origin of the variable illumination pattern is tied to the inner disk, and time domain monitoring of protostellar and protoplanetary systems provides important and unique constraints on the structure of the inner disks around protostars and pre-main sequence stars.

Acknowledgements. We would like to thank the anonymous referee for their help ful comments that improved the quality of the manuscript. B.T.C. and J.J.T. wish to thank L. Hartmann and N. Calvet for suggesting the collaboration while B.T.C. was studying at Leiden University. We acknowledge the support from the University of Michigan to the MDM observatory and the University of Virginia in their support of the Fan Mountain observatory. We thank H. Borsich for helping JJT get started observing on FanCam, as well as A. Mead and J. Heartly for helping with the observations. The National Radio Astronomy Observatory is a facility of the National Science Foundation operated under cooperative agreement by Associated Universities, Inc.

\section{References}

Andre, P., Ward-Thompson, D., \& Barsony, M. 1993, ApJ, 406, 122 Andrews, S. M., Wilner, D. J., Zhu, Z., et al. 2016, ApJ, 820, L40
Arce, H. G., Shepherd, D., Gueth, F., et al. 2007, Protostars and Planets V (Tucson: University of Arizona Press), 245

Aso, Y., Ohashi, N., Aikawa, Y., et al. 2017, ApJ, 849, 56

Blandford, R. D., \& Payne, D. G. 1982, MNRAS, 199, 883

Bontemps, S., Andre, P., Terebey, S., \& Cabrit, S. 1996, A\&A, 311, 858

Connelley, M. S., Hodapp, K. W., \& Fuller, G. A. 2009, AJ, 137, 3494

Facchini, S., Juhász, A., \& Lodato, G. 2018, MNRAS, 473, 4459

Fuente, A., Alonso-Albi, T., Bachiller, R., et al. 2006, ApJ, 649, L119

Guilloteau, S., \& Dutrey, A. 2008, Ap\&SS, 313, 95

Hubble, E. P. 1917, ApJ, 45, 351

Kanneganti, S., Park, C., Skrutskie, M. F., et al. 2009, PASP, 121, 885

Ke, T. T., Huang, H., \& Lin, D. N. C. 2012, ApJ, 745, 60

Loomis, R. A., Öberg, K. I., Andrews, S. M., \& MacGregor, M. A. 2017, ApJ, 840,23

Lubow, S. H. 1992, ApJ, 398, 525

Maloney, P. R., Begelman, M. C., \& Pringle, J. E. 1996, ApJ, 472, 582

Matsumoto, T., Machida, M. N., \& Inutsuka, S.-i. 2017, ApJ, 839, 69

Maury, A. J., André, P., Hennebelle, P., et al. 2010, A\&A, 512, A40

Morales-Calderón, M., Stauffer, J. R., Hillenbrand, L. A., et al. 2011, ApJ, 733 50

Muzerolle, J., Calvet, N., Hartmann, L., \& D’Alessio, P. 2003, ApJ, 597, L149

Muzerolle, J., Furlan, E., Flaherty, K., Balog, Z., \& Gutermuth, R. 2013, Nature, 493, 378

Nixon, C., \& King, A. 2016, in Lect. Notes Phys., eds. F. Haardt, V. Gorini, U. Moschella, A. Treves, \& M. Colpi (Berlin: Springer Verlag), 905, 45

Ohashi, N., Hayashi, M., Ho, P. T. P., \& Momose, M. 1997, ApJ, 475, 211

Ohashi, N., Saigo, K., Aso, Y., et al. 2014, ApJ, 796, 131

Oya, Y., Sakai, N., Lefloch, B., et al. 2015, ApJ, 812, 59

Rebull, L. M., Stauffer, J. R., Cody, A. M., et al. 2015, AJ, 150, 175

Sakai, N., Oya, Y., Sakai, T., et al. 2014, ApJ, 791, L38

Seale, J. P., \& Looney, L. W. 2008, ApJ, 675, 427

Segura-Cox, D. M., Harris, R. J., Tobin, J. J., et al. 2016, ApJ, 817, L14

Sheehan, P. D., \& Eisner, J. A. 2017, ApJ, 851, 45

Snell, R. L., Loren, R. B., \& Plambeck, R. L. 1980, ApJ, 239, L17

Spalding, C., Batygin, K., \& Adams, F. C. 2014, ApJ, 797, L29

Tobin, J. J., Hartmann, L., Calvet, N., \& D’Alessio, P. 2008, ApJ, 679, 1364

Tobin, J. J., Hartmann, L., \& Loinard, L. 2010, ApJ, 722, L12

Tobin, J. J., Hartmann, L., Chiang, H.-F., et al. 2012, Nature, 492, 83

Tobin, J. J., Hartmann, L., Chiang, H.-F., et al. 2013, ApJ, 771, 48

Torres, R. M., Loinard, L., Mioduszewski, A. J., \& Rodríguez, L. F. 2007, ApJ, 671,1813

van 't Hoff, M. L. R., Tobin, J. J., Harsono, D., \& van Dishoeck E. F. 2018, A\&A, 615, A 83

Watson, A. M., \& Stapelfeldt, K. R. 2007, AJ, 133, 845

Whitney, B. A., Wood, K., Bjorkman, J. E., \& Wolff, M. J. 2003, ApJ, 591, 1049

Whitney, B. A., Robitaille, T. P., Bjorkman, J. E., et al. 2013, ApJS, 207, 30

Wolf, S., Schegerer, A., Beuther, H., Padgett, D. L., \& Stapelfeldt, K. R. 2008, ApJ, 674, L101

Wood, K., Smith, D., Whitney, B., et al. 2001, ApJ, 561, 299

Zhu, Z. 2019, MNRAS, 483, 4221 